\title{
The Information from Medical Data Based: Prevalence and Expected Median Survival Time of Drug-Induced Hepatotoxicity Among Thai Patients with TB
}

\author{
Santisith KHIEWKHERN ${ }^{\mathrm{a}, 1}$, Nuchnapa PRATUMCHAI ${ }^{\mathrm{a}}$, Parichart SATTAYARAK ${ }^{\mathrm{a}}$, \\ Patcharin PHUWILERT ${ }^{\mathrm{a}}$, Supattra NOO-IN ${ }^{\mathrm{a}}$ and Naree AREERUK ${ }^{\mathrm{b}}$ \\ ${ }^{a}$ Faculty of public health, Mahasarakham University, Thailand \\ ${ }^{\mathrm{b}}$ Phichit hospital, Phichit Province, Thailand
}

\begin{abstract}
Background: Hepatotoxicity is very frequent and is a dangerously adverse effect of anti-TB medications. This effect can reduce the effectiveness of the treatment by compromising treatment regimens. Among these first-line quadruple therapy drugs (INH, RMP, PZA, and EMB), INH, RMP, and PZA are metabolized mostly by the liver, and due to this, are likely hepatotoxic. However, the survival times of hepatotoxicity among patients with TB in Thailand are currently not available. The aims of the present study were to assess the prevalence and survival time of drug-induced hepatotoxicity in patients with TB. Methods: A cross-sectional retrospective study was performed to explore the survival time of the development of drug-induced hepatotoxicity among 327 patients with TB who received standard drug treatment at the TB clinic in Phichit Hospital. Data was collected from the HOSxP program and medical records from 2016 to 2018. Kaplan-Meier and Cox's regressions were used for data analysis. Results: The results showed that prevalence of drug-induced hepatotoxicity was $6.42 \%$ and confirmed that patients with TB who were $<50$ years of age will be a median survival time on drug-induced hepatotoxicity is 17 days and 30 days for those who age group $\geq 50$ years. Conclusion: The median survival time of drug-induced hepatotoxicity among patients with TB who were $<50$ years of age is 17 days. So, patients with $\mathrm{TB}$ whose ages are less than 50 years should receive liver function tests such as AST and ALT and investigate risk behavior before receiving the antiTB treatment.
\end{abstract}

Keywords. Drug-induced Hepatotoxicity, Tuberculosis, Standard anti-TB drug regiment

\section{Introduction}

The standard treatment regimen for new patients undergoing TB treatment follow the Thailand NTP guidelines 2012 [1] using 2HRZE/4HR [isoniazid (H), rifampin (R), Pyrazinamide (Z), Ethambutol (E)] integrated with the short course therapy for highly effective elimination of TB. However, the most significant problem for patients who received this standard treatment regimen was hepatotoxicity. Subsequently, the patient had to stop their treatment or pause for a long time. This problem influenced drug resistance and death rates from severe hepatotoxicity in patients with TB.

${ }^{1}$ Corresponding Author, Santisith Khiewkhern, Faculty of Public Health, Mahasarakham University, Thailand; 44150. E-mail: santisith.k@msu.ac.th. 
However, the survival times of drug-induced hepatotoxicity development among patients with TB in Thailand are currently not available. The aim of the present study was to assess the prevalence and survival time of drug-induced hepatotoxicity development in patients with TB at one of the general hospitals in the north of Thailand.

\section{Objectives}

The aims of the present study were to assess the prevalence of drug-induced hepatotoxicity in patients with $\mathrm{TB}$ and to explore survival time of drug-induced hepatotoxicity development in patients with TB.

\section{Methods}

3.1 Study design: A cross-sectional retrospective study was used to assess the prevalence of drug-induced hepatotoxicity in patients with TB and to explore survival time of drug-induced hepatotoxicity development in patients with TB who received standard drug regiment.

3.2 Population: The population included all new patients with TB who attended the TB clinic at Phichit Hospital, Phichit Province, Thailand from October $1^{\text {st }}, 2016$ to September $30^{\text {th }}, 2018$.

3.3 Sample: 327 patients with active TB who were on the standard drug regimen at the TB clinic of Phichit Hospital, which is the north of Thailand, from 2016 to 2018.

3.4 Inclusion criteria: Inclusion criteria comprised new confirmed cases with TB who received the standard TB regimen.

3.5 Exclusion criteria: Exclusion criteria were TB patients who were MDR, XDR, XXDR, TDR, had an ALP level > 3 times the UNL, had cirrhosis, chronic liver disease, bile duct cancer, liver cancer, were referred from another hospital, or did not receive continual treatment.

3.6 Outcome measures: Drug-induced hepatotoxicity cases were identified by an expert team at any time after the start of ATT. The criteria were as follows: increased AST or ALT levels of more than 3 times UNL with some hepatotoxicity symptoms such as being bored of food, being squeamish, vomiting, or jaundice; patients who have increased AST or ALT levels of more than 5 times the UNL without hepatotoxicity symptoms; or patients who have increased total bilirubin in the blood of more than 2 times the UNL without increased AST or ALT levels. [2,3]

- Non-Survivors, or events, of this study were patients who develop druginduced hepatotoxicity.

- Survivors, or censored cases, of this study were TB patients with normal, patients who died from other causes, did not follow up, and/or developed liver cancer. 
3.7 Data collection: This study used secondary data from the HOSxP electronic medical record program and manual medical records.

3.8 Statistical analysis: Descriptive statistics were used to describe the characteristics of the subjects. The median survival time of drug-induced hepatotoxicity development at mean of covariates were estimated by Cox 's regressions and Kaplan-Meier survival analysis.

3.9 Ethic Declarations: This study was approved by the Ethics Committee on Human Rights Related to Humans, Phichit Hospital (PH-001/2557).

\section{Results}

A total of 327 patients with TB were newly registered in 2016 and subsequent followup visits until 2018 Most of them, 209 (63.90\% ), were men. The mean age was $51 \pm 17.13$ years. Twenty-one cases ( 11 males, 10 females) developed hepatotoxicity. The prevalence of drug-induced hepatotoxicity was $6.42 \%$. Among patients aged $50+$ years, there were 175 cases $(53.50 \%)$. Twelve cases $(3.67 \%)$ developed hepatotoxicity. The mean age was $51 \pm 17.13$ years, $59.05 \pm 18.67$ years in the hepatotoxicity group and $50.92 \pm 16.93$ in no hepatotoxicity group. Fifty $(39.20 \%)$ cases had a BMI $<18.50$ $\mathrm{Kg} / \mathrm{m}^{2}, 4$ cases $(3.12 \%)$ had hepatotoxicity.

These survival analyses were including age, sex, type of TB, alcohol consumption and Nutrition status as covariates, in addition, drugs and blood parameters were adjusted for these analyses. Finally, 327 patients were included to survival analysis, 21 patients $(6.42 \%)$ developed drug-induced hepatotoxicity. Median survival time of drug-induced hepatotoxicity development was 30 days after they were received standard drug regiment for anti-TB (table 1, Figure 1).

\section{Survival time of drug-induced hepatotoxicity}

Table 1. Mean and Median for Survival time of drug-induced hepatotoxicity.

\begin{tabular}{|c|c|c|c|c|c|c|c|}
\hline \multicolumn{9}{|c|}{ Means and Medians for Survival Time } \\
\hline \multicolumn{4}{|c|}{ Mean $^{\mathrm{a}}$} & \multicolumn{4}{c|}{ Median } \\
\hline Estimate & \multirow{2}{*}{ Std. Error } & $95 \%$ Confidence Interval & \multirow{2}{*}{ Estimate } & \multirow{2}{*}{ Std. Error } & \multicolumn{2}{|c|}{$95 \%$ Confidence Interval } \\
\cline { 3 - 5 } & & Lower Bound & Upper Bound & & & Lower Bound & Upper Bound \\
\hline 44.524 & 10.274 & 24.388 & 64.660 & 30.000 & 5.007 & 20.186 & 39.814 \\
\hline
\end{tabular}

a. Estimation is limited to the largest survival time if it is censored

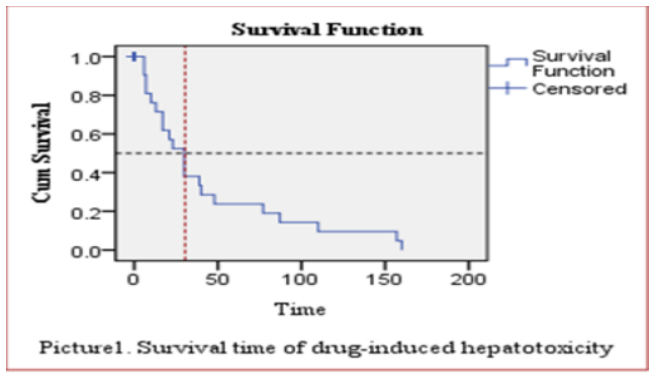

Figure 1. Kaplan-Meier survival analysis of drug-induced hepatotoxicity. 
This survival analysis was separated to two groups by age less than 50 years and $\geq 50$ years. The result showed that median of survival time of drug-induced hepatotoxicity development among TB patients in age group $<50$ years was 17 days after they received standard drug regimen for anti-TB. However, this results show median of drug-induced hepatotoxicity development among TB patients in age group $\geq 50$ years was 30 days after they were received standard drug regiment for anti-TB (table 2, Figure 2).

Table 2. Mean and Median for Survival time of drug-induced hepatotoxicity.

\begin{tabular}{|c|c|c|c|c|c|c|c|c|}
\hline \multicolumn{9}{|c|}{ Means and Medians for Survival Time } \\
\hline \multirow[t]{3}{*}{$50<=1,50>=2$} & \multicolumn{4}{|c|}{ Mean $^{a}$} & \multicolumn{4}{|c|}{ Median } \\
\hline & \multirow[b]{2}{*}{ Estimate } & \multirow[b]{2}{*}{ Std. Error } & \multicolumn{2}{|c|}{$95 \%$ Confidence Interval } & \multirow[b]{2}{*}{ Estimate } & \multirow[b]{2}{*}{ Std. Error } & \multicolumn{2}{|c|}{$95 \%$ Confidence Interval } \\
\hline & & & Lower Bound & Upper Bound & & & Lower Bound & Upper Bound \\
\hline 1 & 32.125 & 11.270 & 10.036 & 54.214 & 17.000 & 5.657 & 5.913 & 28.087 \\
\hline 2 & 52.154 & 15.028 & 22.700 & 81.608 & 30.000 & 9.586 & 11.211 & 48.789 \\
\hline Overall & 44.524 & 10.274 & 24.388 & 64.660 & 30.000 & 5.007 & 20.186 & 39.814 \\
\hline
\end{tabular}

a. Estimation is limited to the largest survival time if it is censored.

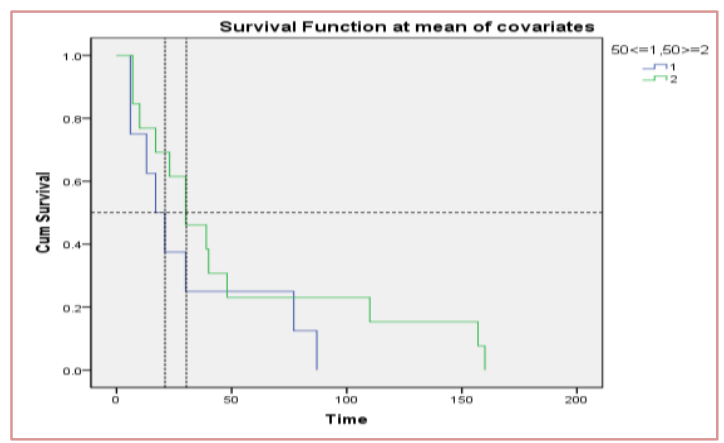

Figure 2. Survival time at mean of covariates

\section{Discussion}

The results showed that the prevalence of drug-induced hepatotoxicity among patients with TB was $6.42 \%$ and confirmed that patients with TB who were $<50$ years of age will be a median survival time on drug-induced hepatotoxicity is 17 days; this result was similar to the retrospective study from a large TB center in the UK [4], which found half $(53 \%)$ of the hepatotoxicity occurred within 2 weeks of starting anti-TB treatment, $87.6 \%$ occurred within 8 weeks and no positive association between increasing age and risk of drug-induced hepatotoxicity because health behaviors which are risk to increase toxins in the blood and collect in the liver such as alcohol consumption, low weight, especially in patients with high drug dosages as patients with HIV-1 co-infection. However, this result was contrasts with a previous prospective case-control study of Singla R. and colleagues [5] and other previous studies [6, 7], which found that an age of $\geq 60$ years was a specific risk factor for hepatotoxicity. So, 
patients with TB who age are less than 50 years should receive liver function tests such as AST and ALT and investigate risk behavior before receiving the TB treatment.

\section{Conclusion}

The prevalence of drug-induced hepatotoxicity among patients with TB was $6.42 \%$. The median survival time of drug-induced hepatotoxicity among patients with TB who were $<50$ years of age is 17 days. So, patients with TB who age are less than 50 years should receive liver function tests such as AST and ALT and investigate risk behavior before receiving the anti-TB treatment.

Acknowledgments: We would like to thank the nurses and doctors at the TB Care Centre in Phichit Hospital, Thailand. The authors also thank Robert Bachtell Eastland for correcting the English in this article. This study was supported by the Faculty of Public Health, Mahasarakham University, and Phichit Hospital, Thailand.

\section{References}

[1] Teschke R, Danan G. Drug-induced liver injury: Is chronic liver disease a risk factor and a clinical issue? Expert Opin Drug Metab Toxicol. 2016;1-14. http://dx.doi.org/10.1080/17425255.2017.1252749.

[2] Khalili H., et al. Anti-tuberculosis drugs related hepatotoxicity; incidence, risk factor, pattern of changes in liver enzymes and outcome. DARU 2009; 17: 163-167.

[3] Vidyasagar Ramappa and Guruprasad P. Aithal. Hepatotoxicity Related to Anti-Tuberculosis Drugs: Mechanisms and Management. Journal of Clinical and Experimental Hepatology. 2013 Mar; 3(1): 3749. Published online 2012 Dec 20. doi: 10.1016/j.jceh.2012.12.001.

[4] Aula Abbara, Sarah Chitty, Jennifer K. Roe, Rohma Ghani, Simon M. Collin, Andrew Ritchie, Onn Min Kon, John Dzvova, Harriet Davidson, Thomas E. Edwards, Charlotte Hateley, Matthew Routledge, Jim Buckley, Robert N. Davidson and Laurence John : Drug-induced liver injury from anti-tuberculous treatment: a retrospective study from a large TB centre in the UK.2016; BMC Infectious Diseases (2017) 17:231: 9-1. DOI 10.1186/s12879-017-2330-z

[5] Singla R., Sharma S.K., Mohan A. Evaluation of risk factors for anti-tuberculosis treatment induced hepatotoxicity. Indian J Med Res. 2010;132:81-86. [PubMed] [Google Scholar]

[6] Bright-Thomas RJ, Gondker AR, Morris J, Ormerod LP. Drug-related hepatitis in patients treated with standard anti-tuberculosis chemotherapy over a 30-year period. Int $\mathrm{J}$ Tuberc Lung Dis. 2016;20(12):1621-4.

[7] Wan Beom Park, Won Kim, Kook Lea Lee, Jae Joon Yim, Moonsuk Kim, Yong Jin Jung, Dong Hea Kim, Yoon Jun Kim,Jung-Hwan Yoon, Myong-don Oh and Hyo Suk Lee. Anti-tuberculosis druginduced liver injury in chronic hepatitis and cirrhosis. Journal of infection 2010; 61:323-29. 